\title{
AN $H$-SPACE WITH FINITE DIMENSIONAL HOMOLOGY WHOSE LOOP SPACE HAS TORSION
}

\author{
RICHARD D. PORTER
}

\begin{abstract}
An example is given of a simply connected $H$-space whose integral homology is zero in dimensions greater than two and such that the integral homology of the loop space has torsion.
\end{abstract}

The $\bmod p$ homology of the example is isomorphic to the $\bmod p$ homology of $S^{3}$ for all primes $p$. The example is not of finite type, and it is not known in general whether the loop space of a finite $H$-space has torsion free integral homology. See Problem 53 in [1].

Let $f: S^{3} \rightarrow K(Q, 3)$ classify the element $\alpha \otimes 1$ in $H^{3}\left(S^{3}: Z\right) \otimes Q \cong H^{3}\left(S^{3}: Q\right)$, where $\alpha$ is the canonical generator of $H^{3}\left(S^{3}: Z\right)$. Construct the fiber square

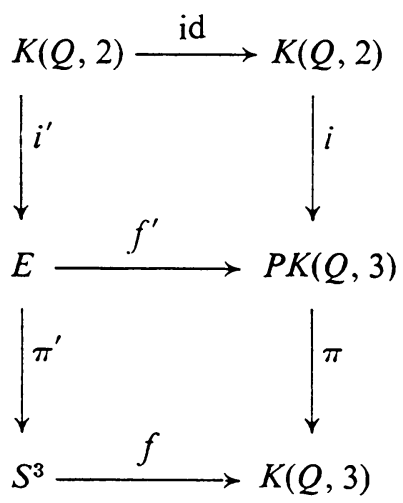

where $P K(Q, 3)$ denotes the path space of the Eilenberg-Mac Lane space $K(Q, 3)$, and $E$ denotes the pullback.

1. $E$ is a simply connected $H$-space with $H_{2}(E: Z)=Q / Z$, and $H_{i}(E: Z)=$ 0 for $i>2 ; H_{1}(\Omega E: Z)=Q / Z$. Further, $\pi_{*}^{\prime}: H_{*}(E: Z / p) \rightarrow H_{*}\left(S^{3}: Z \mid p\right)$ is an isomorphism for all primes $p$.

Proof. $\alpha \otimes 1$ is a primitive element of $H^{3}\left(S^{3}: Q\right)$, so we can assume that $f$ is a map of $H$-spaces. Thus $E$ is an $H$-space. The homology of $E$

Received by the editors April 18, 1972.

AMS (MOS) subject classifications (1970). Primary 57F25; Secondary 55F20.

Key words and phrases. $H$-space, fiber square, Eilenberg-Mac Lane space, pullback, divided polynomial algebra.

(c) American Mathematical Society 1973 
is obtained by calculating the Serre spectral sequence of the fibration $K(Q, 2) \rightarrow^{i^{\prime}} E \rightarrow \rightarrow^{\pi^{\prime}} S^{3}$. The spectral sequence is a spectral sequence of Hopf algebras since the maps $i^{\prime}$ and $\pi^{\prime}$ are maps of $H$-spaces.

Note. $\quad \tilde{H}_{*}(K(Q, 2): Z) \cong \tilde{\Gamma}(x) \otimes Q$ where $\tilde{\Gamma}(x)$ denotes the elements of positive dimension in the divided polynomial algebra over $Z$ on one generator $x$ of dimension 2. $H_{1}(\Omega E: Z)=Q / Z$ since $\pi_{1}(\Omega E)=Q / Z$. The result concerning $\pi_{*}^{\prime}$ follows from the observation that $\tilde{H}_{*}(K(Q, 3): Z / p)=$ 0 .

From the universal coefficient theorem for cohomology, it follows that $H^{3}(E: Z)=\hat{Z}$ (the completion of $Z$ ), and $H^{i}(E: Z)=0$ for $i \neq 0,3$.

\section{REFERENCE}

1. Problems presented to the 1970 AMS Summer Colloquium in Algebraic Topology, edited by R. James Milgram, Proc. Sympos. Pure Math., vol. 22, Amer. Math. Soc., Providence, R.I., 1971, pp. 187-201.

Department of Mathematics, Brown University, Providence, Rhode Island 02912 